\title{
Der Ätna und seine Pflanzenwelt
}

\section{Hilke Steinecke \& Peter Schubert}

\begin{abstract}
Mount Etna on Sicily is the highest active volcano of Europe at more than 3000 meters. Various volcanic phenomena can be observed within a relatively small area. The flora at the different altitudes is enormously diverse. This article describes the vegetational zones and presents a choice of wildflowers including several endemic plants.
\end{abstract}

\section{Zusammenfassung}

Der Ätna auf Sizilien ist der höchste und aktivste Vulkan Europas. Auf kleinem Raum können verschiedene vulkanische Phänomene beobachtet werden. Die Pflanzenwelt am Ätna ist aufgrund der großen Höhenunterschiede sehr abwechslungsreich. Die Vegetationsstufen sowie dort vorkommende Wildpflanzen, darunter auch einige Endemiten des über $3000 \mathrm{~m}$ hohen Vulkans, werden vorgestellt.

\section{Der Ätna}

Mit rund $3300 \mathrm{~m}$ Höhe ist der Ätna (italienisch Etna) höher als Deutschlands höchster Berg, der Zugspitze. Werden die Kanaren mit dem Teide auf Teneriffa nicht zu Europa gezählt, ist der Ätna der höchste und gleichzeitig auch der aktivste Vulkan Europas. Seit 2013 ist der Ätna mit seiner einzigartigen Natur ein UNESCOWeltnaturerbe.
Von vielen Richtungen aus betrachtet, ob vom Nebrodi-Gebirge auf Sizilien oder von den Bergen Süd-Kalabriens, der Ätna erhebt sich majestätisch und überragt mit seinem lange schneebedeckten Gipfel die sizilianische Landschaft. Als Schichtvulkan (Stratovulkan) ist er ähnlich wie Japans höchster Vulkan Fuji gleichmäßig kegelförmig geformt mit steilen Flanken.

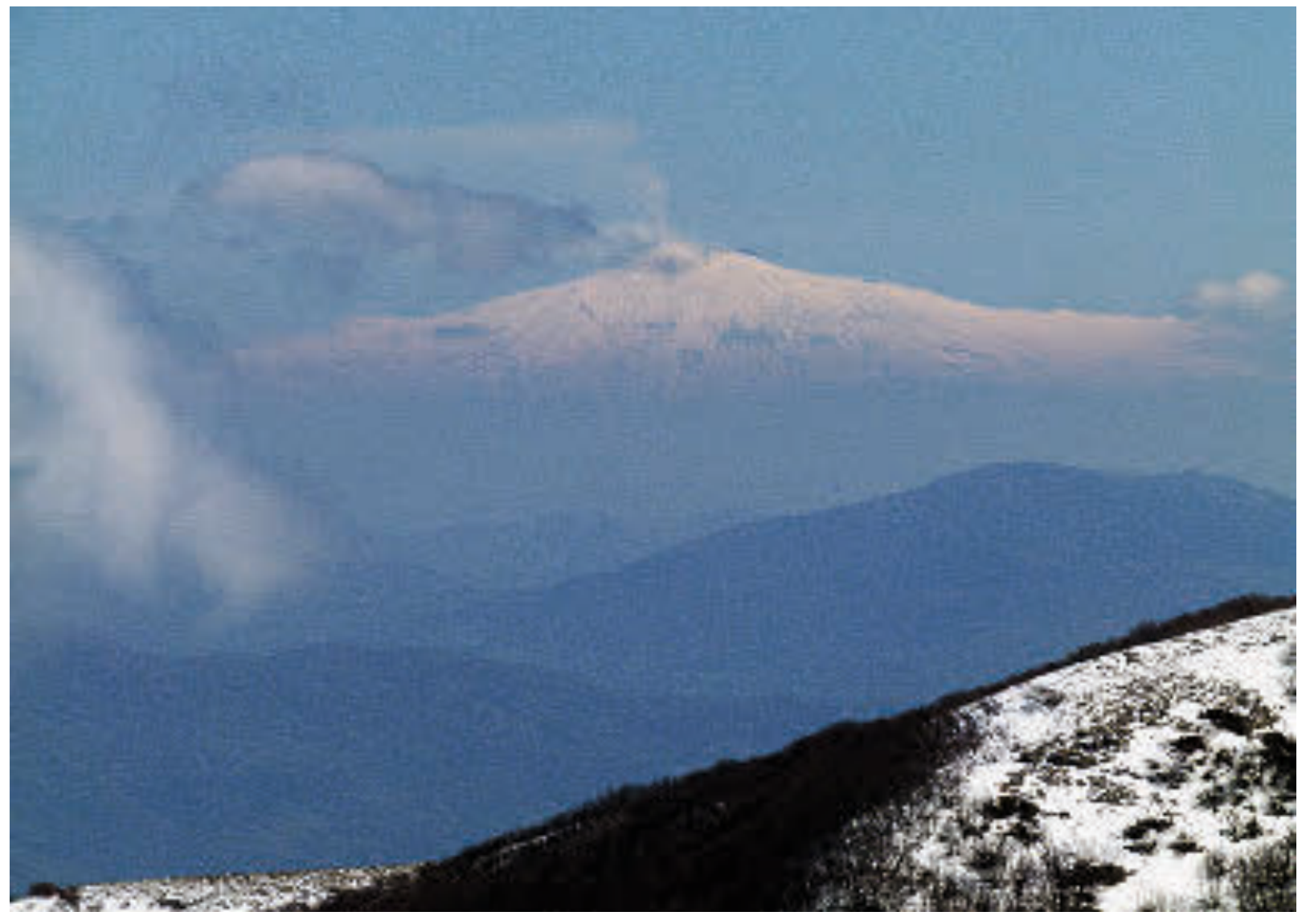


Der Ätna ist wie der Vesuv und die nördlich von Sizilien gelegenen Liparischen Inseln in einer Region entstanden, wo die afrikanische und die europäische Kontinentalplatte aufeinanderstoßen bzw. sich die afrikanische Platte unter die europäische schiebt. An diesen sogenannten Hotspots kommt es häufig zu Vulkanismus.

Vor etwa 600000 Jahren begann sich der Ätna erstmals zu bilden, der eigentliche Stratovulkan entstand allerdings erst vor 100000 Jahren. Durch Ausbrüche und Erosion hat sich der Ätna immer wieder verändert. Die älteren Teile des heutigen Vulkans sind nur rund 10000 Jahre alt, und die Bereiche des heute noch aktiven Teils sind noch einmal einige Jahrtausende jünger. Bis heute kommt es regelmäßig zu Ausbrüchen, die keine oder meist vergleichsweise geringe Schäden hinterlassen. Bei größeren Ausbrüchen haben in vergangenen Zeiten aber oft die Menschen darum gebetet, dass ihre am Ätna gelegenen Ortschaften nicht von der Lava überrollt werden. So wurden im Jahr 1923 viele Häuser in Linguaglossa durch einen Lavastrom aus der Nähe des Monte Nero zerstört.

Im Jahre 2002 fiel die Seilbahn auf den Ätna einem stärkeren Ausbruch zum Opfer. In der Nähe der auf 1900 m Höhe gelegenen Hochebene Piano Provenzana, heute Ausgangspunkt für viele Wanderungen am Ätna-Nord, kann man noch Reste eines verschütteten Hotels entdecken und auf einer alten Straßenkurve entlangwandern, die in einem erkalteten Lavastrom endet. Ein spektakuläres Naturschauspiel lieferte jüngst der starke Ausbruch im Dezember 2015.

Die Eruptionen des Ätnas finden nicht nur an den vier Gipfelkratern statt, häufig treten

Abb. 1 (Seite 12): Ätna, vom Nebrodi-Gebirge aus betrachtet.

Abb. 2 (oben): Ein vom Lavastrom 2002/2003 verschüttetes Haus.

Abb. 3 (Mitte): Kraterlandschaft an den Flanken des Ätnas.

Abb. 4 (unten): Lavaflüsse sammeln sich häufig im Bovetal.
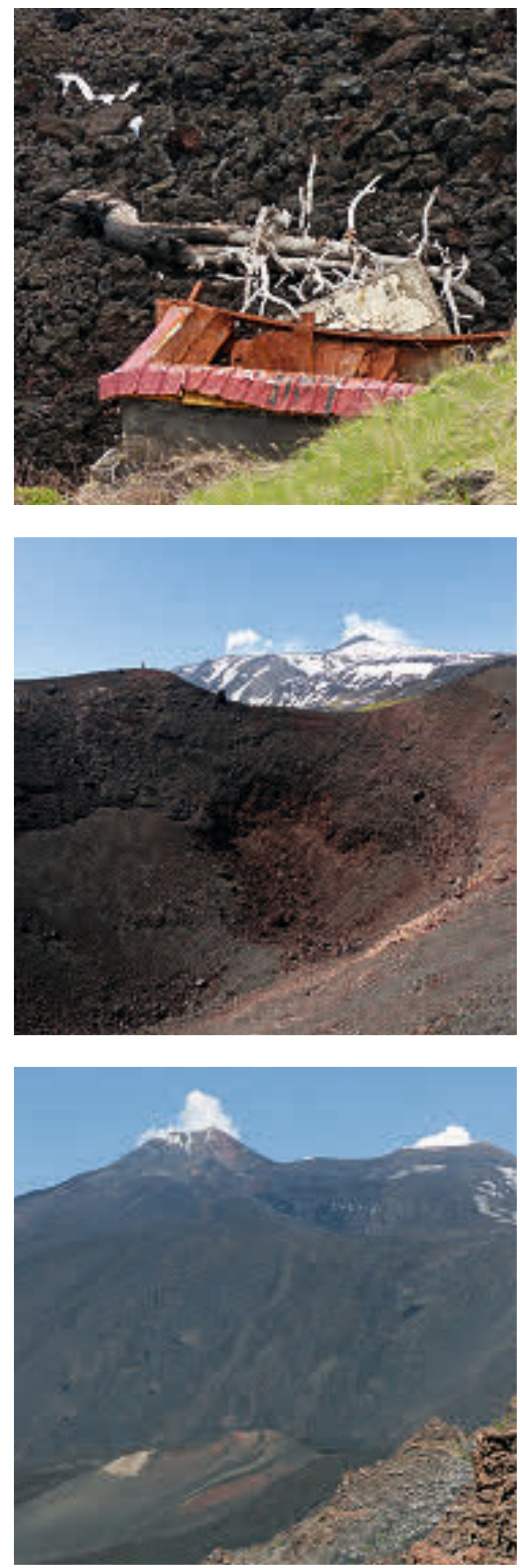

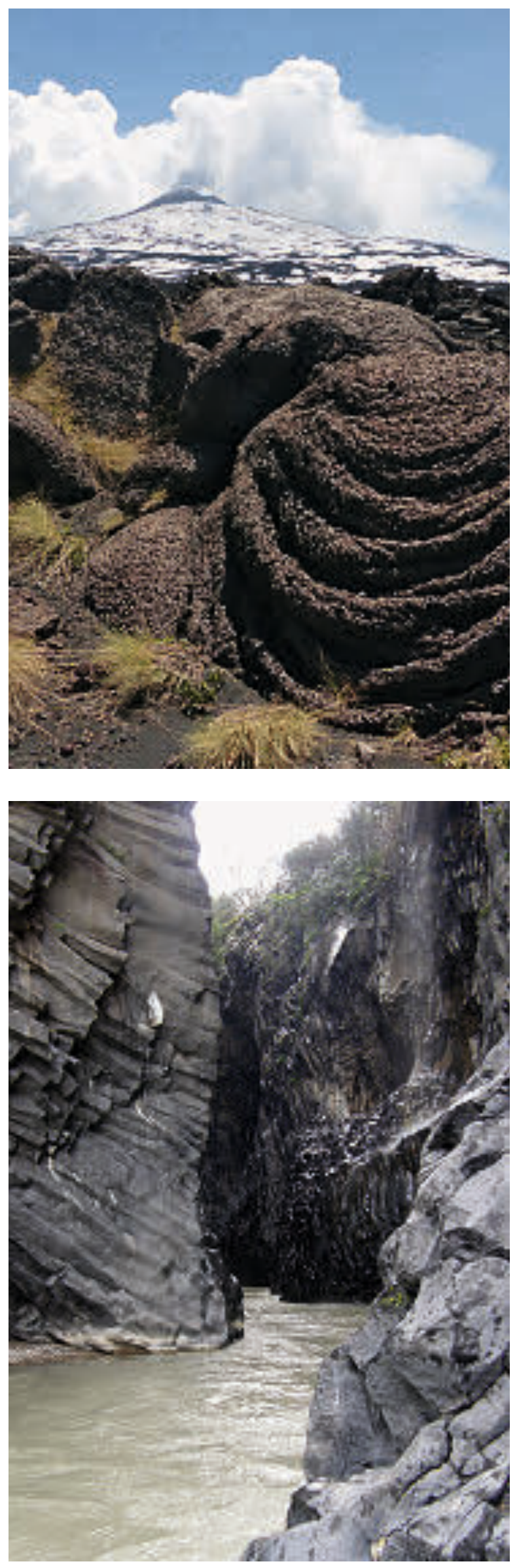

Lava und Gase auch an den Flanken aus, es entstehen regelmäßig neue Krater, Aschekegel und Eruptionsspalten. Die vulkanische Aktivität an den Flanken des Ätnas hat bisher etwa 200-300 kleinere Krater hinterlassen. Beeindruckend ist das breite Bovetal (Valle de Bove) am Südosthang des Ätnas mit seinem Talgrund im Westen auf $1700 \mathrm{~m}$ Höhe. Seine Entstehung ist noch unklar, vermutlich geht es auf einen $\mathrm{Zu}$ sammenbruch eines Einsturzkraters (Caldeira) eines Vorgängervulkans sowie auf Hangrutschungen zurück. Vor etwa 8000 Jahren brach die Ostwand des Bovetals ab. Die abgerutschten Gesteinsmassen lösten im Ionischen Meer wohl einen sehr starken Tsunami aus, der weite Küstenabschnitte in Griechenland und Nordafrika verwüstete. Die frühen Bewohner Siziliens verließen ihre zerstörte Insel und besiedelten daraufhin Malta.

In das Tal ergießen sich häufig während der Eruptionen breite Lavaströme. Blicke von oben auf das Tal lassen sehr gut den Verlauf der Lavaströme auch noch nach dem Erkalten erkennen. Eine derartige Lavawüste wie heute gab es hier nicht immer. Noch im 18. und 19. Jh. gab es hier Hochweiden, daher der Name Val de Bove (= Ochsental).

Die basische und kieselsäurearme Lava des Ätnas ist relativ dünnflüssig. Dadurch können aus ihr die Gase leicht entweichen. Entsprechend baut sich kein Druck auf, der zu einer größeren Explosion führen könnte. Der Ätna ist deshalb ein relativ harmloser Vulkan, dessen Aktivitäten recht gut eingeschätzt werden können. Es gibt kaum einen durch Messstationen so gut datenmäßig aufgearbeiteten Vulkan wie

Abb. 5 (oben): Stricklava am Ätna-Nord.

Abb. 6 (unten): Alcantara-Schlucht mit schönen Basaltsäulen.

Abb. 7 (Seite 15 oben): Ein kleiner Schlammvulkan (Vulcanello) in der Nähe von Agrigent.

Abb. 8 (Seite 15 Mitte): Ätna-Ginster fällt durch seine gelblichgrünen Triebe auf.

Abb. 9 (Seite 15 unten): Eine von noch mehreren älteren Flaum-Eichen am Ätna. 
den Ätna. Der Vesuv dagegen, äußerlich scheinbar ruhend, kann im Innern einen großen Druck aufbauen. Wenn es irgendwann zu einem explosiven Ausbruch kommen sollte, würde dieser vermutlich viel gefährlicher sein als am Ätna. Hinzu kommt, dass rund um den Vesuv ein mit Neapel viel dichter als am Ätna besiedeltes Gebiet bedroht würde.

Wenn dünnflüssige Lava oberflächlich abkühlt und aushärtet, darunter die Lava aber noch weiterfließt, entstehen Lavahöhlen. Am Ätna gibt es einige eindrucksvolle, große Lavahöhlen, die teilweise auch zu besichtigen sind.

Faszinierend am Ätna ist, dass auf kleinstem Raum verschiedenste vulkanische Erscheinungsformen relativ einfach und vor allem oft gut zugänglich erkundbar sind. Aschekegel, Vulkantrichter, Stricklava oder Vulkanbomben sind allgegenwärtig.

Klassische Basaltformationen sind z. B. mühelos in der küstennahen Alcantara-Schlucht (Gole dell'Alcantara) am Fuße des Ätnas zu bestaunen. Ein Erdbeben hat hier ein altes Basaltfeld mit gleichmäßigen sechseckigen Basaltsäulen in zwei Teile zerrissen. Dazwischen konnte sich dann der Alcantara-Fluss einen Weg suchen. Der eindrucksvolle Canyon ist bis $25 \mathrm{~m}$ hoch und im Extremfall nur $2 \mathrm{~m}$ breit.

Die kleinen Schlammvulkane (Vulcanelli) bei Agrigent sind ein Phänomen des sog. kalten Vulkanismus. In Verbindung mit unter Druck stehendem Wasser (meist im Bereich tektonischer Schwachstellen) wird tonreiches Sedimentgestein aufgeschlämmt und es bilden sich Strukturen, die an Minivulkane erinnern. Die Vulcanelli waren über viele Jahre hinweg eine Touristenattraktion, bis das Betreten Ende 2014 verboten wurde, nachdem es durch einen $20 \mathrm{~m}$ hohen Schlammausbruch zu einem tödlichen Unfall gekommen war.

\section{Nutzpflanzen und Kulturland der mediterranen Stufe}

Aufgrund der großen Höhenunterschiede ist rund um den Vulkanberg eine deutliche Vegetationszonierung mit unterschiedlichen Pflanzen anzutreffen (Marchese 1991). Die Lavaböden des Ätnas sind sehr fruchtbar, sodass sich
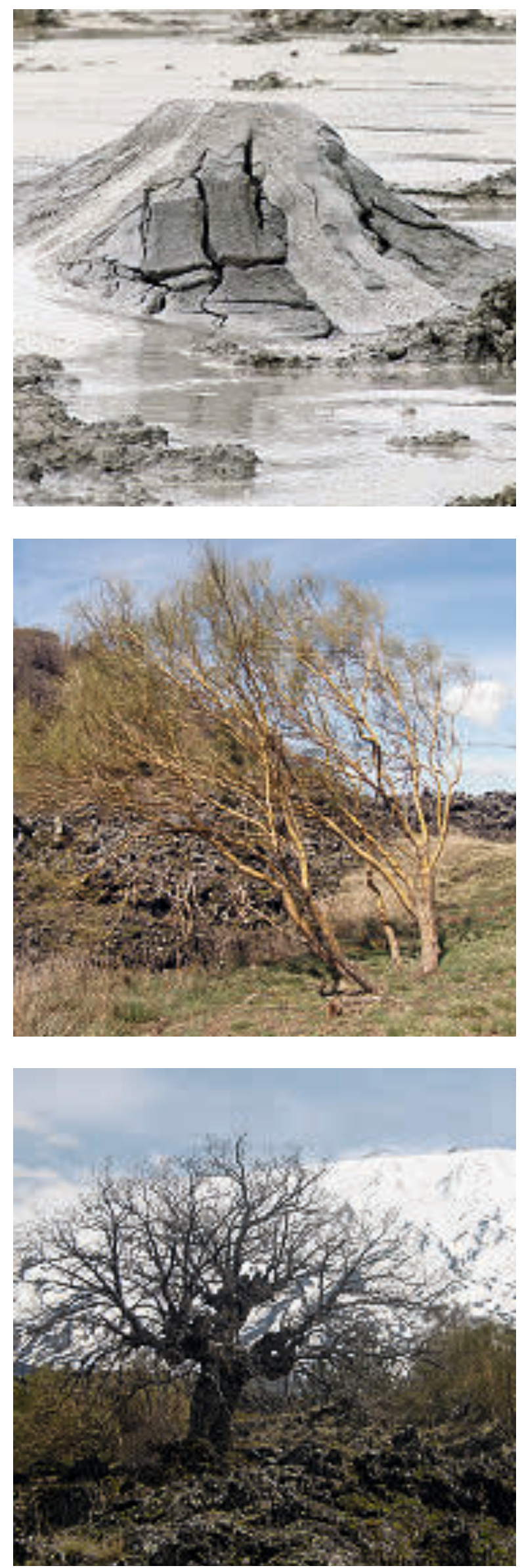


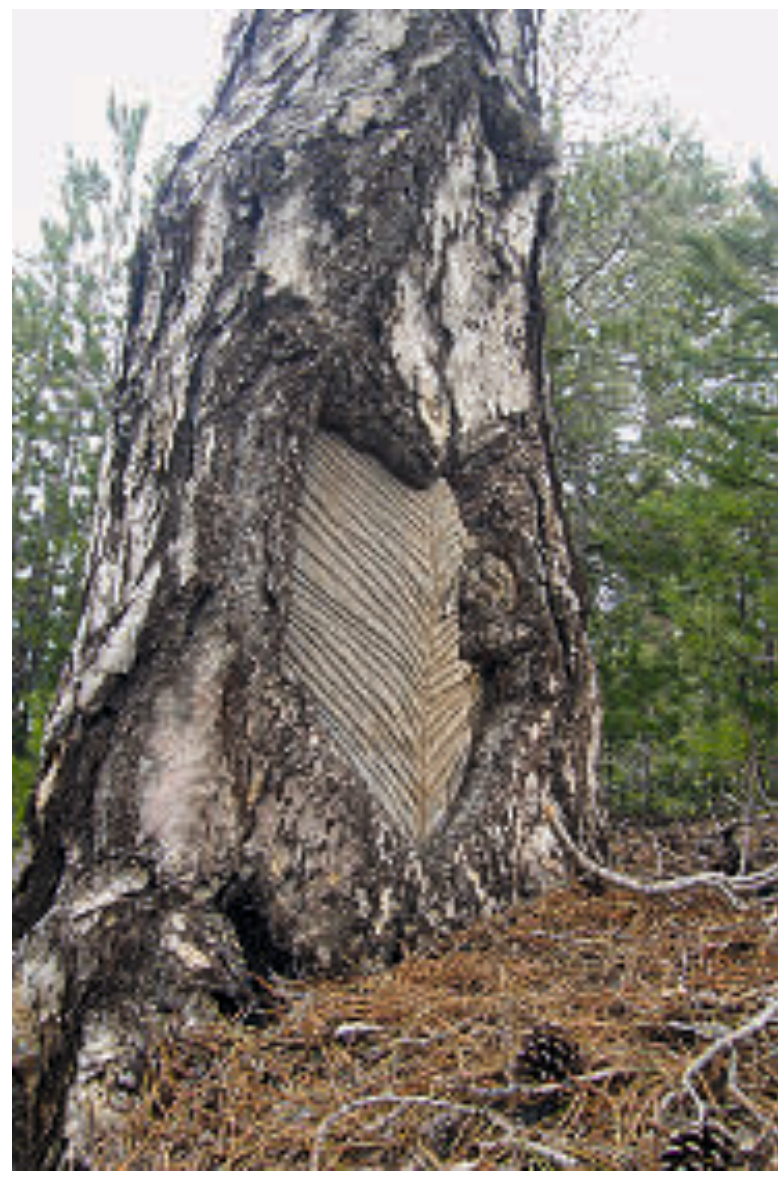

in der unteren mediterranen Stufe der Anbau von Kulturpflanzen anbietet. Durch die intensive Landwirtschaft wurde die natürliche Vegetation an den unteren Hängen des Ätnas (Macchie, z. B. mit Pistacia lentiscus) weitgehend verdrängt. Küstennah wurden zahlreiche Zitrusplantagen angelegt. Auf den landwirtschaftlich genutzten Flächen haben Weinbau und Olivenkultur große Bedeutung. Der kleine Ort Bronte an den Westhängen des Ätnas ist für den Anbau von hochwertigen Pistazien berühmt. Auf dem rauen und z. T. steilen vulkanischen Gelände sind die Bearbeitung der Pflanzungen und die Ernte mit Maschineneinsatz nicht möglich, alles ist deshalb Handarbeit. Die hier geernteten Früchte gelten als die besten Pistazien weltweit. In Bronte wird jährlich im Herbst das Pistazienfest (Sagra del Pistacchio) gefeiert. Der Pistazienanbau wurde schon vor 1000 Jahren durch die Araber in diese Region Siziliens gebracht. In den niedrigeren Lagen gedeihen außer vielen Nutzpllanzen auch diverse Zierpflanzen, darunter auch stattliche Kameli- en, die man in dem einen oder anderen Hausgarten entdecken kann.

An Gehölzen gedeihen in den niedrigen Lagen vor allem wärme- und trockenheitsliebende Arten wie die immergrüne Stein-Eiche (Quercus ilex) sowie Hopfenbuche (Ostrya carpinifolia) und Manna-Esche (Fraxinus ornus). Ein bedeutender Nutzbaum ist die Esskastanie (Castanea sativa), die hier seit Jahrhunderten in weitläufigen Hainen in den mittleren Lagen angepflanzt wird. Wenige alte und große Exemplare sind touristische Anziehungspunkte; am Ätna steht auf rund $700 \mathrm{~m}$ Höhe in der Nähe von Sant'Alfio die uralte, mittlerweile auseinandergebrochene und aus drei Hauptstämmen bestehende Kastanie der hundert Pferde, die bereits Goethe bestaunt hat. Angeblich fanden während eines Unwetters 100 Pferde mitsamt Reitern in diesem Baum Unterschlupf. Dieser Baum gilt nach Meinung einiger Dendrologen als der älteste Baum Europas. In den Kastanienhainen blühen im Frühling reichlich Orchideen (z. B. Dactylorhiza romana) und Alpenveilchen (Cyclamen repandum). Immer wieder reißen Lavaströme, die regelmäßig bei kleineren oder größeren Eruptionen entstehen, Schneisen in den Bergwald. Während der Wald diese Wunden kaum oder nur sehr langsam schließen kann, ist der Ätna-Ginster schnell zur Stelle. Er ist auf Lavaströmen vom Fuß des Ätnas bis hin zur Baumgrenze anzutreffen und gehört zu den ersten Besiedlern der rohen Lavaböden. An manchen Stellen haben die einzelnen Ginster-Pflanzen zueinander einen relativ großen Abstand. Das ist vor allem dort wichtig, wo die einzelnen Individuen auf dem porösen, im Sommer staubtrockenen Lavaboden gegenseitig zu stark um Wasser konkurrieren würden. Besonders reichliche und kräftige Exemplare

Abb. 10 (oben): Durch Einritzen der Stämme wurde früher Kiefernharz gewonnen.

Abb. 11 (Seite 17 oben): Gerippe von Schwarz-Kiefern, die durch den Ausbruch von 2002/2003 zerstört wurden.

Abb. 12 (Seite 17 unten): Die Ätna-Birke hat einen besonders weiß leuchtenden Stamm. 


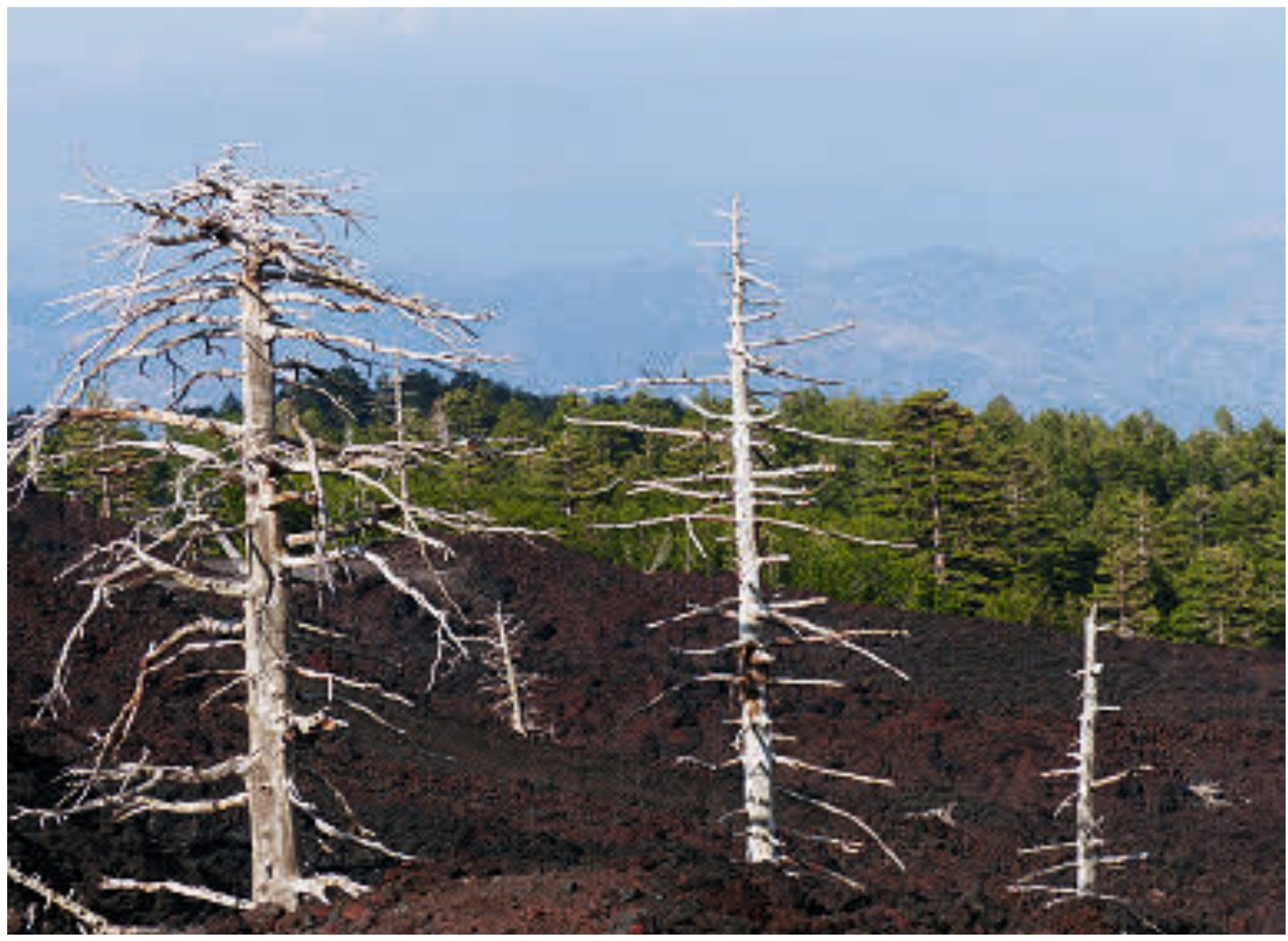

kann man auf Wanderungen oberhalb von Bronte, also an den Nordwesthängen des Ätnas, erkunden. Markantes Merkmal der nicht blühenden Exemplare ist die gelblichgrüne Rinde, die zur Photosynthese fähig ist. Daneben gehören zu den ersten Besiedlern der Lava natürlich diverse Kryptogamen wie Moose und Flechten (z. B. Stereocaulon vesuvianum).

\section{Supramediterrane Stufe}

\section{mit sommergrünen Wäldern}

In Höhen zwischen etwa 1000 und $1500 \mathrm{~m}$ gedeihen zwischen den Lavaströmen Wälder aus sommergrünen Eichen wie Flaum-Eichen (Quercus pubescens) und Zerr-Eichen (Q. cerris), die auch hier oben an einigen Stellen mit Kastanien durchmischt sind. Am Ätna gibt es noch insgesamt 56 knorrige, sogenannte tausendjährige Eichen (Flaum-Eichen). Da sie zunehmend von Schwarz-Kiefern bedrängt wurden, initiierten Umweltschützer ein Projekt, diese Bäume freizuschneiden und zu retten. Im zeitigen Frühling fällt an lichten Standorten Euphorbia

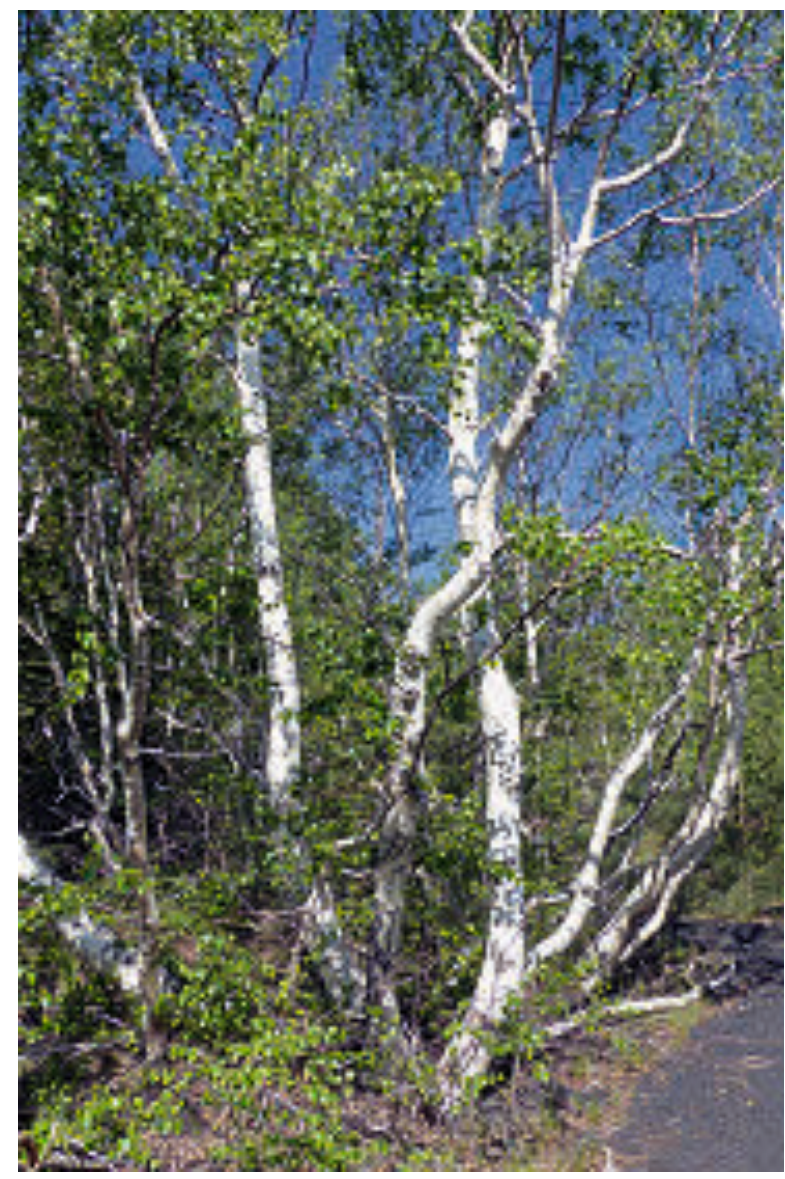



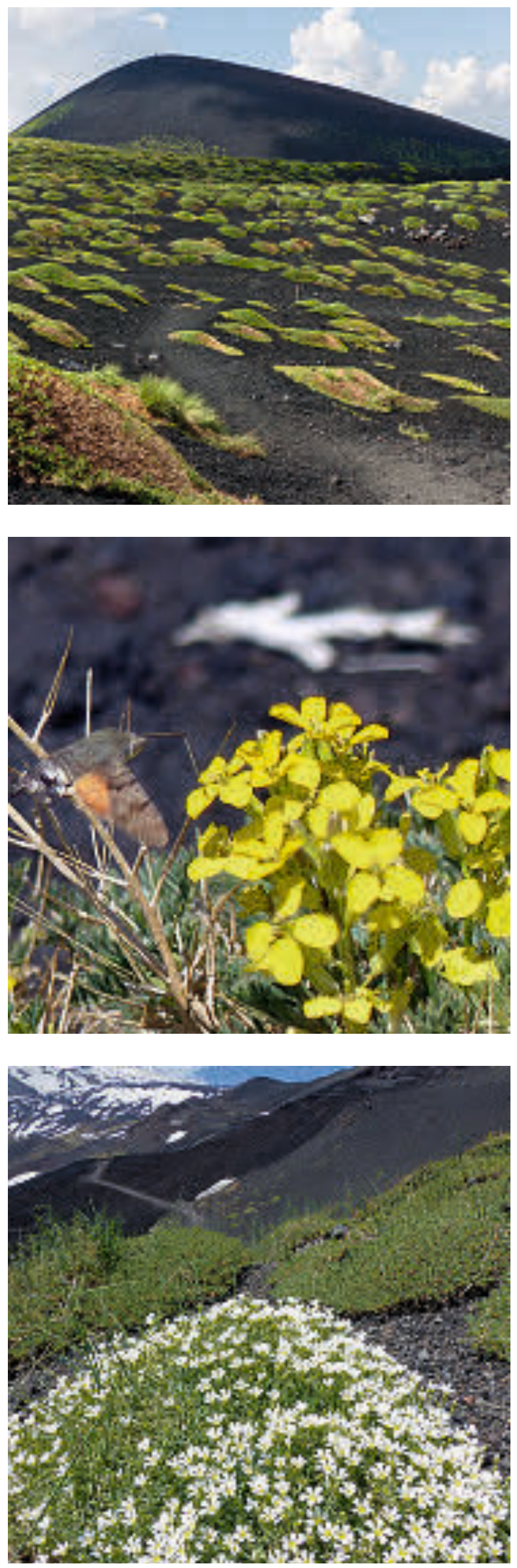

rigida mit ihren leuchtend gelben Hoch- und Nektarblättern auf.

\section{Mediterrane alpine Stufe}

Weiter höher bis etwa $2000 \mathrm{~m}$ schließen sich Wälder mit Korsischen Schwarz-Kiefern (Pinus nigra subsp. laricio), Buchen, Eichen und der endemischen Ätna-Birke (Betula aetnensis) an. Die z.T. sehr großen, alten Kiefern bilden an manchen Stellen üppige Bestände (Pineta di Linguaglossa und Serra la Nave).

Für die Einheimischen waren die SchwarzKiefern früher wichtige Nutzbäume, da sie am Stamm angeritzt wurden, um das aus der Wunde heraustropfende Harz aufzufangen. An manchen Bäumen sind noch heute alte Ritzwunden anzutreffen, die noch nicht ganz überwallt sind. Für die Harzsammler war es immer wieder ein unwiederbringlicher Verlust, wenn Teile des alten Kiefernwaldes durch Eruptionen und Lavaströme zerstört wurden. Es ist ein bizarres, fast unwirkliches Bild, wenn am NordÄtna oberhalb der Piano Provenzana weiße Skelette alter Kiefern mitten in der Lava stehen. Die Stämme verwittern nur sehr langsam. Die hier erwähnten Kiefern verbrannten während des Ausbruches von 2002/2003, stehen hier also schon über 10 Jahre. Wenn umgestürzte Kiefernstämme von Lava überrollt werden, dabei verbrennen und anschließend die Lava darüber aushärtet, bilden sich Hohlräume in der Form eines Kiefernstammes, die man an manchen Stellen entdecken kann. Die Kiefern am Ätna sind sehr robust und hitzetolerant. Manchmal teilen sich Lavaströme auf ihrem Weg ins Tal. Auf den kleinen zurückgebliebe-

Abb. 13 (oben): Igelpolsterflur mit Astracantha sicula oberhalb der Baumgrenze.

Abb. 14 (Mitte): Erysimum bonnanianum mit Taubenschwänzchen.

Abb. 15 (unten): Cerastium aetneum bildet dichte Polster.

Abb. 16 (Seite 19 oben): Das Ätna-Veilchen bildet oberhalb der Baumgrenze z. T. üppige Bestände.

Abb. 17 (Seite 19 unten): Anthemis aetnensis oberhalb des Bovetals. 
nen Vegetationsinseln zwischen den beiden (einst glühend heißen) Armen des Lavastromes sind dann die Kiefern erhalten geblieben, obwohl die Hitze auch hier sicherlich sehr hoch war.

Zwischen den Kiefern und noch etwas höher gedeiht vor allem an den Nord- und Ostflanken des Vulkans die 4-15 m hohe endemische Ätna-Birke (Betula aetnensis; nach Auffassung mancher Botaniker ein Synonym unserer Betula pendula). Ihre schneeweißen Stämme reflektieren die Strahlung und schützen im Sommer vor Überhitzung auf dem sich schnell erwärmenden schwarzen Lavaboden. Ätna-Birken gehören auch mit zu den ersten Besiedlern junger Lavafelder am Ätna. Sie müssen winterliche Kälte, Schnee, Schneebruch, sommerliche Hitze und Trockenheit ertragen können. Ende Mai entfalten die Bäume ihr frisch grünes Laub.

\section{Altimediterrane Stufe}

Diese Vegetationsstufe zieht sich von der Waldgrenze bis auf ca. 3000 m Höhe. Je nach Exposition und Feuchtigkeit ist die Vegetation auf den verschiedenen Seiten des Ätnas unterschiedlich dicht ausgeprägt. Während der von Touristen wegen der Seilbahn intensiv besuchte Ätna-Süd nur sehr karg bewachsen ist, gedeiht am feuchteren Ätna-Nord eine typische Igelpolsterflur aus niedrigen, stechenden Pflanzenkissen, die in relativ großem Abstand zueinander wachsen. An diesen durch Vulkanismus, schneereiche Winter und trocken-heiße Sommer geprägten Extremstandorten können nur noch Spezialisten existieren. Fast alle Arten, die man hier antrifft, sind Endemiten. Viele von ihnen tragen den Artbeinamen aetnensis o. ä.

Die markanteste Art ist hier sicherlich der endemische igelpolsterförmige Tragant (Astragalus aetnensis = Astracantha sicula), der von 1700-2450 m in der Lava teils üppige Bestände bildet. Wie eine Schafherde wirken die oft über einen Meter breiten grünen Kissen auf dem dunklen Untergrund. Die Polsterform schützt die Pflanze vor Kälte, übermäßiger Austrocknung und Wind. Im Italienischen heißt die Art Spini Santi, Dornen der Heiligen. Die Polster sind extrem stachelig. Im Schutz dieser
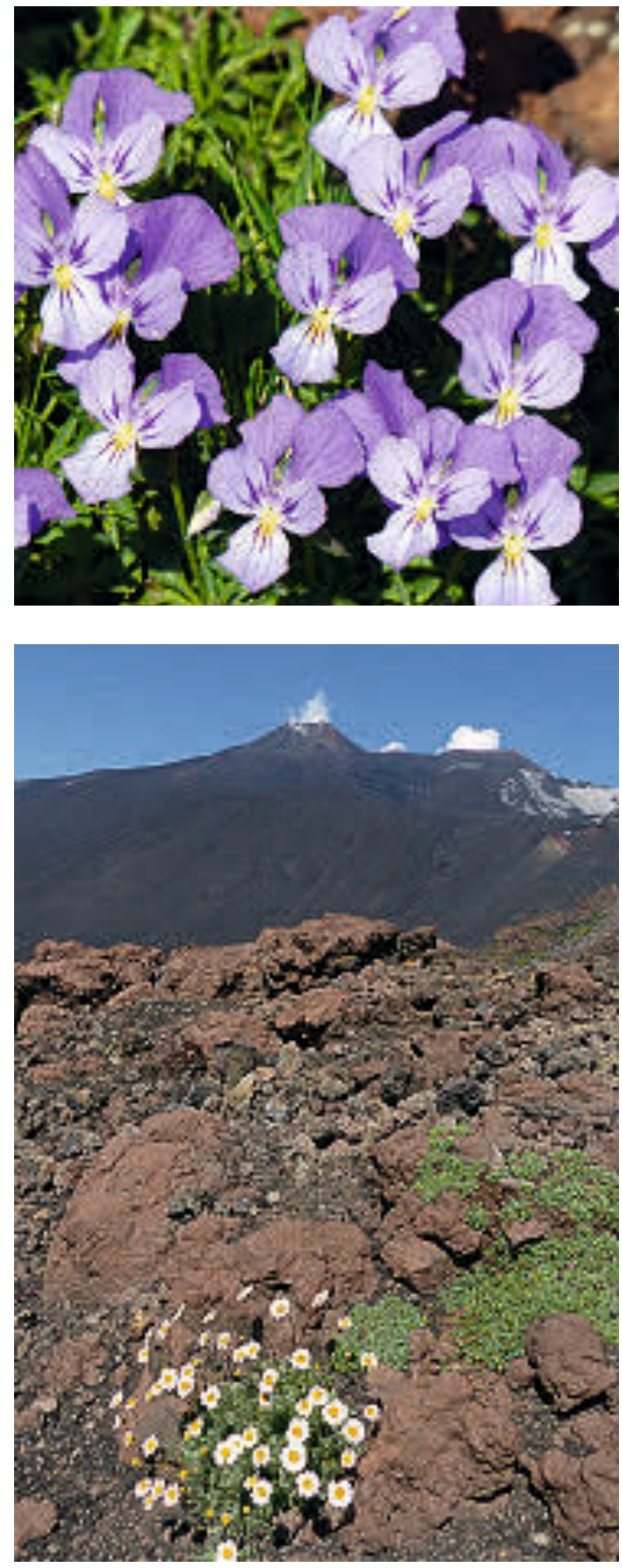

Polster siedeln sich oft auch noch verschiedene andere Pflanzen an. $\mathrm{Zu}$ diesen Arten gehören z.B. Ätna-Berberitze (Berberis aetnensis) und Ätna-Seifenkraut (Saponaria sicula). In der Höhe blüht es Ende Mai/Anfang Juni erstaunlich üppig. So sind der endemische gelblütige Schöterich (Erysimum bonnanianum) und das 

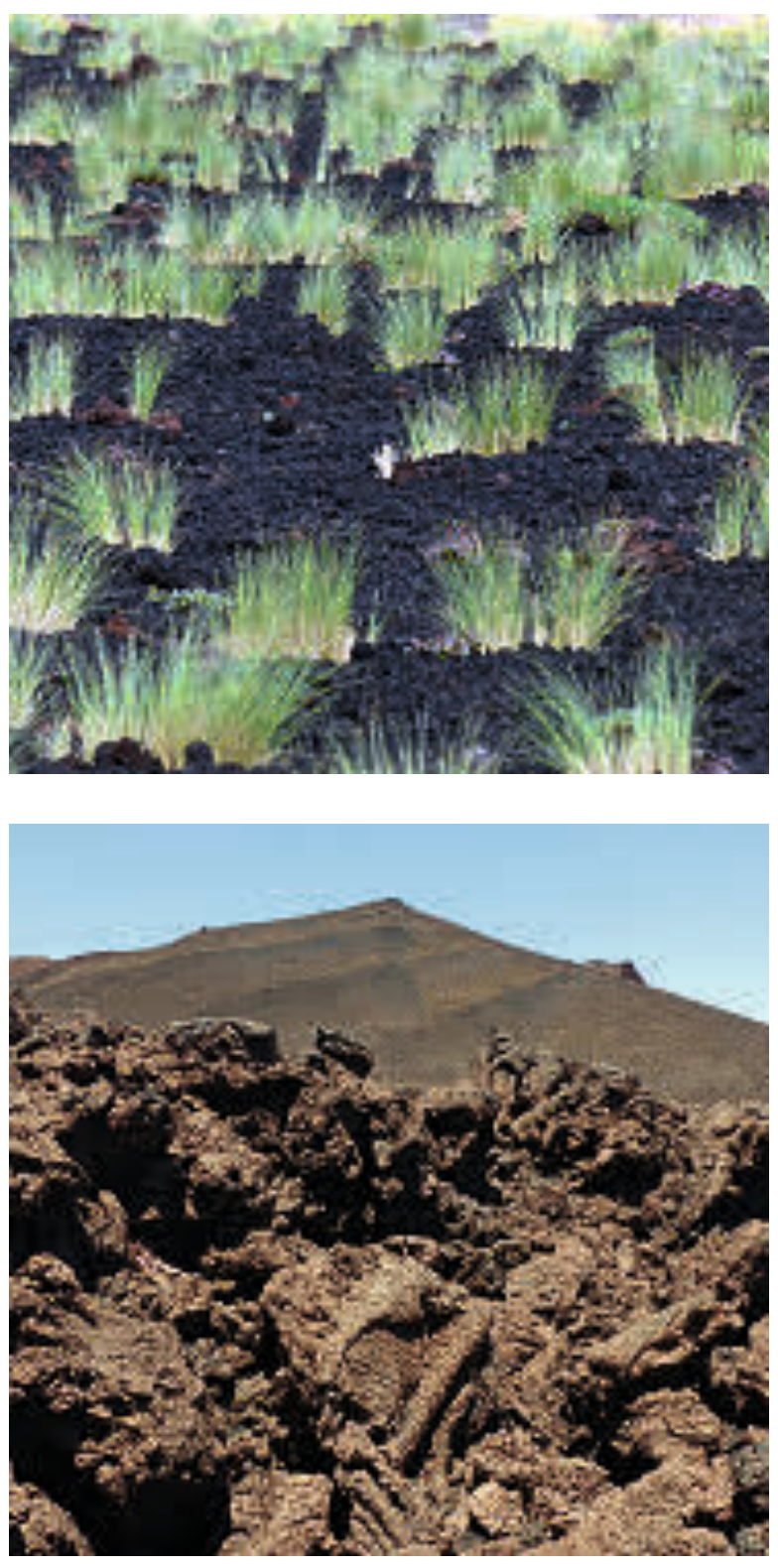

endemische Hornkraut (Cerastium aetneum = C. tomentosum var. aetneum) wichtige Futterpflanzen für viele nektarsuchende Insekten wie Distelfalter, Kleiner Fuchs und Taubenschwänzchen. Das endemische Ätna-Veilchen (Viola aethnensis) kommt bis $2800 \mathrm{~m}$ Höhe vor, bildet z. T. große Trupps in Reinkultur oder ist mit den Tragant-Polstern verwoben. Seine Blüten sind sehr variabel, ihre Farben können Weiß, Hellblau Blauviolett oder sogar Gelb sein. Eine der am höchsten steigenden Gefäßpflanzen am Ätna ist die Ätna-Margerite (Anthemis aetnensis), die bis in Höhen von $3050 \mathrm{~m}$ im Gipfelbereich nachgewiesen wurde. Ebenfalls an Extremstandorten bis $3000 \mathrm{~m}$ Höhe gedeiht eine auffällige gelbe Komposite (Robertia taraxacoides). Wo Lavaschotter und -sand leicht ins Rutschen kommen, können sich vor allem diejenigen Arten gut halten, die das Überschütten leicht überstehen (z.B. Rumex scutatus f. aetnensis) oder über Rhizome ,wandern" können. An manchen Stellen sieht man zwischen den Igelpolstern des Tragants einzelne Grasbüschel von Poa aetnensis (= Bellardiochloa variegata subsp. aetnensis $=$ Poa variegata), die wie ausgesät in Reihen auftreten, weil sie entlang eines Rhizoms entstanden sind.

Die Blütezeit in der altimediterranen Zone zieht sich über mehrere Wochen hin, da nicht alle Arten gleichzeitig blühen. Um Hornkraut, Schöterich und Ätna-Veilchen zu sehen, sollte man Ende Mai oder Anfang Juni am Ätna sein. Der Ätna-Ginster, der bei uns als Zierpflanze etwa im Mai blüht, öffnet seine Blüten je nach Standort und Höhenlage erst von Juni bis August. Auch Tragant sowie Seifenkraut sind an ihren höchsten Standorten erst etwa im August blühend anzutreffen. Das Botanisieren am Ätna lohnt sich also zu verschiedenen Zeiten. Ein idealer Standort für Quartiere, von denen man zu Wanderungen am Ätna-Nord aufbricht, ist Linguaglossa.

\section{Aschewüste am Gipfel}

Über 3000 m Höhe gibt es am Ätna so gut wie keine Pflanzen mehr. Vulkanische Aktivität, Ausstoß von Asche, Lava und giftigen Dämpfen machen hier das Leben so gut wie unmöglich. Zudem ist der Gipfel für viele Monate von Schnee bedeckt, was ebenso die Entwicklung einer Vegetation beeinträchtigt. Mitunter liegt um Ostern noch so viel Schnee, dass man rund um die Bergstation der Seilbahn in $2500 \mathrm{~m}$ Höhe durch freigefräste Hohlwege mit noch 2-3 m hohen Schneewänden wandern kann.

Abb. 18 (oben): Poa aetnensis vermehrt sich mit Hilfe von Rhizomen, deshalb die reihige Anordnung der Pflanzen.

Abb. 19 (unten): Vegetationsfreie Lavawüste.

Abb. 20 (Seite 21): Winterlandschaft am Ätna-Süd mit Blick auf den Gipfelkraterbereich. 


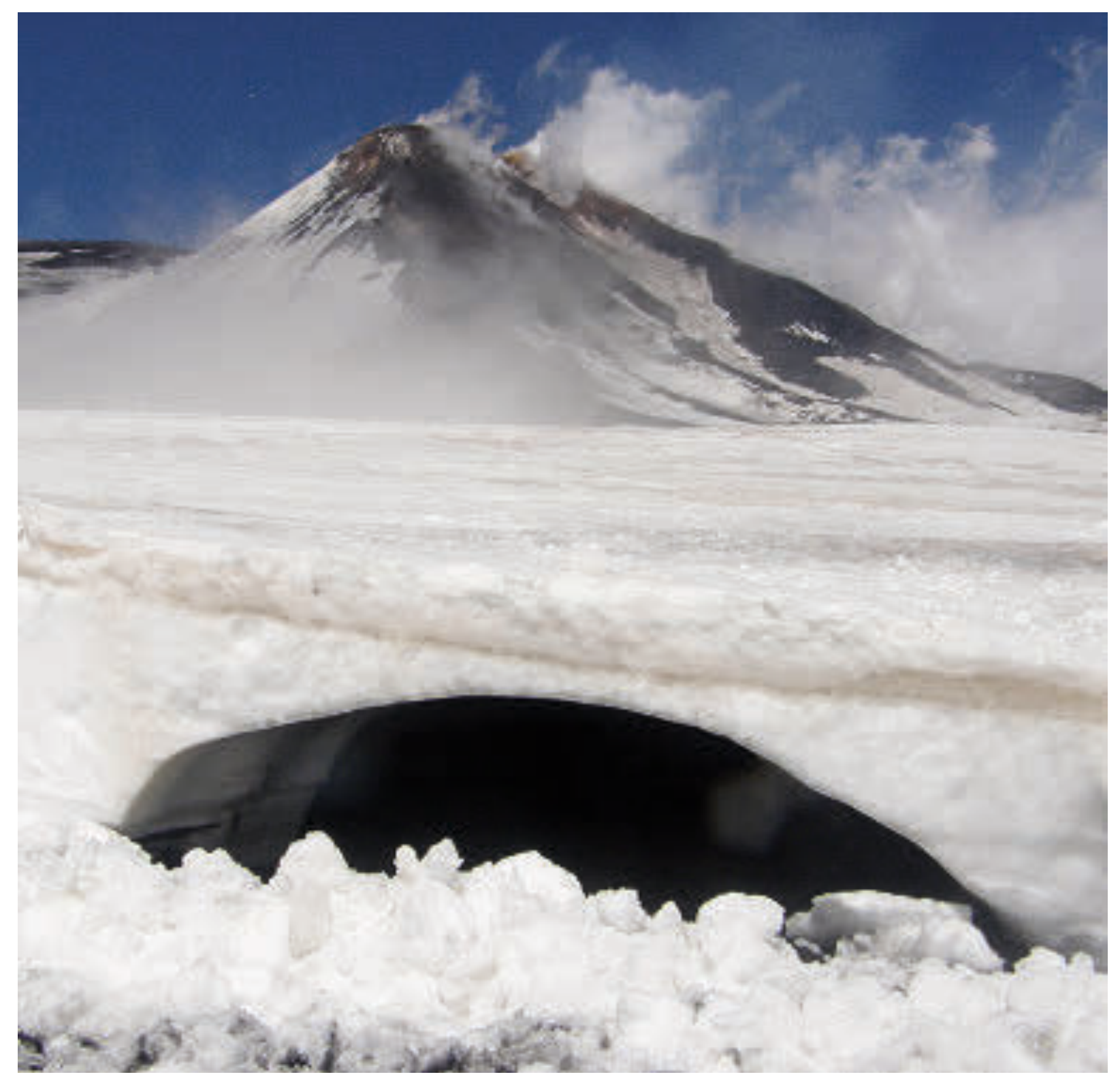

Auf der Nordseite hält sich der Schnee naturgemäß deutlich länger, ein Aufstieg zum Gipfel von der Nordseite her ist deshalb meist erst $a b$ Ende Juni möglich.

Der Ätna ist für den Botaniker ein extrem lohnendes Ziel. Auf kleinem Raum können hier eine deutliche Vegetationszonierung sowie viele Endemiten erkundet werden. Landschaftlich ist der Ätna äußerst reizvoll. Durch den aktiven Vulkanismus verändert er sich ständig, sodass er von einem auf ein anderes Jahr seine Gestalt, auch im markanten Gipfelbereich, durchaus verändern kann und dabei z. B. neue Krater gebildet werden. Jeder Besuch des Ätna kann folglich für neue Überraschungen sorgen.

\section{Literatur}

Marchese, E. P. 1991: Piante e fiori dell'Etna. - Palermo.

\section{Internetseiten}

Flora e Vegetazione dell'Etna

http://www.dipbot.unict.it/ctnatura/vulcano/ETN_IND. html

Etna natura

http://www.etnanatura.it/foto/argomentophp?dir=Fiori\& descr=Fiori 